\section{Gibt es eine altersabhängige Korrelation zwischen CRP-Spiegel und Demenzrisiko?}

In mittleren Lebensjahren fördern erhöhte CRP-Spiegel den geistigen Verfall. Doch im fortgeschrittenen Alter scheint sich die Bedeutung des Entzündungsmarkers zu wenden. Bei über 75-Jährigen, die keine kognitiven Defizite aufweisen, könnte ein hoher CRP-Spiegel ein Zeichen für eine entsprechend günstige familiäre Veranlagung sein.

chon in früheren Studien war ein $\mathrm{Zu}$ sammenhang zwischen erhöhten CRP-Spiegeln und verminderten kognitiven Fähigkeiten nur bei Probanden bis zu 63 Jahren, nicht aber bei älteren Menschen beobachtet worden. Bei über 75-Jährigen mit intakter Kognition waren höhere CRP-Werte in einer Studie sogar mit einer besseren Gedächtnisleistung assoziiert. Offenbar spiegeln hohe CRPWerte bei dieser Personengruppe eine genetische Veranlagung für das Altern ohne geistigen Abbau wider, wie Ärzte um Dr. Jeremy M. Silverman von der Mount Sinai School of Medicine in New York herausgefunden haben.

Die Mediziner hatten von 277 Männern im Alter ab 75 und ohne kognitive Beeinträchtigung Eltern und Geschwister per AD Risk Questionnaire untersucht. Das Ergebnis: Das Risiko der Verwandten für eine Demenzerkrankung - insgesamt waren 40 von 1.329 Personen betroffen - lag umso niedriger, je höher der CRPSpiegel des geistig fitten Bruders beziehungsweise Sohnes war. Je nachdem, ob der CRP-Spiegel in das untere, mittlere oder obere CRP-Tertil fiel, waren von den Verwandten im Alter von 90 Jahren $13 \%$, $12 \%$ oder nur $5 \%$ dement geworden. Dieselbe Korrelation, sogar in noch stärkerer Ausprägung, fanden die New Yorker Ärzte bei 202 erstgradigen Verwandten von 51 geistig unversehrten Personen im Alter von 85 Jahren aufwärts. Hohe CRPWerte bei Personen mit „erfolgreichem kognitiven Altern“ sind damit, so Silverman und Kollegen, „ein phänotypisches Merkmal" für ein familiäres, also genetisch bedingtes erfolgreiches kognitives Altern.

Aber wie kommt es, dass ein Merkmal, das in mittleren Lebensjahren einen Risikofaktor darstellt, später im Leben mit einem nützlichen Effekt verknüpft ist? Vorstellbar ist, dass die schädliche Wirkung durch einen anderen (genetisch

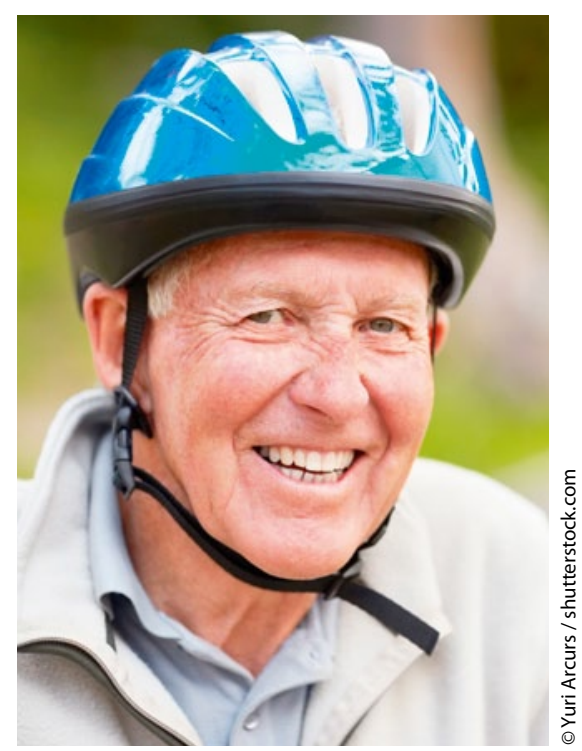

Menschen über 75 Jahren mit erhöhten CRP-Spiegeln könnten eine Veranlagung für geringen geistigen Abbau haben. es mehr AU-Tage durch psychisch Erkrankte.
$\mathrm{D}$ er Krankenstand der AOK-Versicherten in Baden-Württemberg ist im ersten Halbjahr 2012 mit 4,9\% auf Vorjahresniveau geblieben. Demnach waren die AOK-versicherten Arbeitnehmer (Pflicht- und freiwillig Versicherte) im Schnitt 7,9 Tage pro Jahr krank geschrieben.

Fortgesetzt hat sich der Trend, dass der Anteil psychisch Erkrankter weiter zugenommen hat. Der Anteil psychischer Erkrankungen sei um 0,6 Prozentpunkte auf $10,2 \%$ aller AU-Tage gestiegen (Vorjahreszeitraum: 9,6\%), so die AOK. Vor zehn Jahren habe dieser Wert bei der Hälfte gelegen. „Vorsorgemöglichkeiten und Behandlungsstrukturen müssen dringend festgelegten) Faktor gepuffert wird. Die Mehrzahl der Menschen mittleren Alters haben diesen Schutzfaktor nicht, das heißt, bei ihnen kommen die nachteiligen Wirkungen eines hohes CRP-Spiegels wie der Anstieg des Risikos für Herz-Kreislauf-Erkrankungen, Demenz und vorzeitigen Tod zum Tragen. Ein hohes Alter erreichen damit vor allem Personen, bei denen ein protektiver Faktor diesen CRPabhängigen Komplikationen gegensteuert. Dadurch ist bei ihnen ein erhöhter CRP-Spiegel wenn auch nicht die Ursache, aber doch das messbare Merkmal einer demenzpräventiven Kondition deren Beschaffenheit es noch zu erforschen gilt.

Alternativ könnte die Assoziation eines hohen CRP mit hoher geistiger Leistungsfähigkeit einem besonders aktiven Immunsystem zu verdanken sein. Es gibt nämlich Hinweise, dass bei betagten Menschen mit intakter Kognition das Immunsystem hochreguliert ist.

Dr. Beate Schumacher

Silverman JM et al. C-reactive protein and familial risk for dementia: A phenotype for successful cognitive aging. Neurology 2012; 79: $1116-23$

\title{
AOK Südwest: Immer öfter streikt die Psyche
}

Der Krankenstand im Südwesten bleibt bei der AOK konstant - allerdings gibt

verändert werden, damit diese Entwicklung gestoppt werden kann", forderte der AOK-Vorstandschef Dr. Christopher Hermann. Er verwies dazu auf den Facharztvertrag, den die Kasse mit Ärzten und Psychotherapeuten geschlossen hat.

Bei $38 \%$ der AU-Tage war eine Langzeiterkrankung von mehr als sechs Wochen die Ursache.

$22,2 \%$ aller AU-Tage entfielen auf Muskel- und Skeletterkrankungen, vor allem Rückenerkrankungen, meldet die Kasse.

Florian Staeck

Quelle: Presseinformation der AOK 